\title{
Tsafon
}

Revue d'études juives du Nord

$72 \mid 2016$

Juifs, Israéliens, dans la littérature française et israélienne

\section{Bible et nature dans le roman Les Innocents d'Assaf Gavron}

Françoise Marti-Delescaut

\section{(2) OpenEdition}

Journals

Édition électronique

URL : https://journals.openedition.org/tsafon/362

DOI : $10.4000 /$ tsafon.362

ISSN : 2609-6420

Éditeur

Association Jean-Marie Delmaire

Édition imprimée

Date de publication : 1 décembre 2016

Pagination : 65-88

ISSN : $1149-6630$

Référence électronique

Françoise Marti-Delescaut, « Bible et nature dans le roman Les Innocents d'Assaf Gavron », Tsafon [En ligne], 72 | 2016, mis en ligne le 31 mai 2018, consulté le 30 juin 2021. URL : http:// journals.openedition.org/tsafon/362 ; DOI : https://doi.org/10.4000/tsafon.362 


\title{
Bible et nature dans le roman Les Innocents d'Assaf Gavron
}

\author{
Françoise Marti-Delescaut ${ }^{*}$
}

Un roman israélien paraît en 2013 et, à double titre, c'est un événement dans la littérature israélienne contemporaine. Il n'adopte pas le ton dépressif, habituel dans la récente littérature hébraïque, et ne fait pas des tentatives expérimentales stylistiques afin d'innover. Son ton est délibérément réaliste : il peint une fresque documentée à la manière de Zola, mais loin d'être polémique, doloriste ou grinçant, il observe d'un regard tendre et amusé ce petit monde que la littérature a jusqu'à présent négligé. De manière inhabituelle, il décrit la vie dans une implantation juive des rives du Jourdain, une de ces «colonies » sauvages tellement contestées, à contrecourant de la bien pensance internationale, à contrecourant même de sa propre sensibilité politique.

Ce roman, Les Innocents (Paris, éd. Rivages, 2014, trad. Laurent Cohen), est un gros livre de 650 pages, paru en hébreu en Israël (TelAviv, Yediot Aharonot, 2013) sous le titre Hagiva, La Colline, titre sans doute plus représentatif de l'enjeu de l'intrigue et de la puissance d'évocation que représente, pour ces implantés religieux, cette colline incomparable qu'ils sont incapables de quitter. C'est ainsi que se trouvent inextricablement mêlés le thème de la Bible, de la religion vécue au quotidien et le thème de la Nature et de la Terre d'Israël qu'ils se sentent légitimes d'occuper. C'est ce lien intime entre ces deux thèmes que nous explorons ici, mais ce roman foisonnant aurait pu offrir bien d'autres pistes d'exploration (la représentation de l'inefficacité des autorités israéliennes, l'humour et l'ironie de l'auteur, la stratégie narrative «à l'américaine », le conflit israélo-palestinien etc.).

\footnotetext{
* Université Charles de Gaulle - Lille 3.
} 


\section{Un roman original}

\section{Définition des termes}

Sans doute, faut-il d'emblée revenir sur ce que nous entendons ici par «Bible» et « Nature ». L'intrigue se déroulant en Terre d'Israël (Eretz Israel) parmi des personnages tous juifs, le mot «Bible» est entendu ici par ce qu'il est d'usage d'appeler l'Ancien Testament, et nous élargirons l'étude à l'ensemble des pratiques religieuses qui en sont indissociables.

Quant au mot « Nature », c'est un terme plus difficile à définir. Si pour le monde moderne, il renvoie presque spontanément au monde « extérieur », le monde physique qui fait l'environnement (minéral, végétal et animal) de l'homme, en hébreu moderne ce mot tev'a, nature ${ }^{1}$, ne se rencontre pas dans la Bible, ni même dans la Mishna, du moins pas dans ce sens (en hébreu biblique, c'est un terme qui évoque l'idée de laisser une marque). Passant par l'idée d'une effigie frappée sur une monnaie (comme Adam façonné à l'image de Dieu), et donc de désigner la nature de l'homme, la racine [TB'] ne se retrouvera qu'aux $\mathrm{XI}^{\mathrm{e}}-\mathrm{XII}{ }^{\mathrm{e}}$ siècles dans le sens moderne de «nature des choses» sous la plume de Juda Halévi. Par ailleurs, notre conception de la nature est héritée de la culture gréco-romaine où les termes phusis et natura renvoient à l'idée de « pousser» (grec) ou de «naître» (latin). Progressivement, le terme hébreu tev'a s'est approprié le sens grec de phusis. Aussi établir des équivalences entre notre conception de la nature et celle du texte biblique présente une certaine difficulté. En effet, dans la Bible, ce qui se rapproche le plus de cette notion, c'est la Création, telle qu'elle est décrite dans le livre de la Genèse, et contenant donc tout ce qui est créé (ciel, terre, astres, végétaux, animaux). Cependant la Bible n'utilise jamais les termes traduits habituellement en français par «Créateur» et «créatures », elle les nomme par leur nom ou utilise les termes génériques [basar] chair et [ma'asim] œuvres. Du reste, le roman n'emploie quasiment pas ce mot, mais, comme la Bible, préfère nommer les éléments (champs, collines, paysages, soleil). C'est nous qui avons choisi ce terme générique, le trouvant assez pratique, tout en connaissant les problèmes de fond qu'il soulève et qui nous dépassent. C'est pourquoi, sans nous y appesantir davantage nous travaillerons en estimant qu'il y a

\footnotetext{
${ }^{1}$ Ces considérations sont tirées de l'article de Jean-Georges Kahn : « Brève histoire de l'idée de nature dans la pensée juive », site du Judaïsme d'Alsace et de Lorraine, http://judaisme.sdv.fr/perso/c-yashar/nature.htm
} 
équivalence entre Création et Nature et donc que la notion sera élargie au cosmos (éléments, astres etc.).

L'étude s'appuie principalement sur les deux personnages centraux du roman: Othniel Assis, responsable de l'implantation, et Gaby Nehoustan.

\section{L'auteur : Assaf Gavron (né en 1968)}

Assaf Gavron est né à Arad dans une famille d'immigrants anglais (son père était journaliste et sa mère enseignante) et il grandit à Motza Illit, près de Jérusalem. Il poursuit des études supérieures en communication à Londres, puis à Vancouver. Ensuite, il travaille comme journaliste en Israël, comme enseignant d'écriture créative à l'Université de Bar Ilan, puis comme producteur. Après avoir vécu avec sa famille à Tel-Aviv, il s'est installé depuis quelques années aux États-Unis, dans le Nebraska, où il enseigne la littérature israélienne à l'Université d'Omaha. C'est un intellectuel de gauche très polyvalent, écrivain, créateur de jeux informatiques, capitaine de l'équipe nationale de football israélienne, traducteur en hébreu de romans anglo-saxons (Jonathan Safran Foer, Salinger, Philip Roth, Rowling etc.); actuellement, il traduit pour Nathalie Portman le scénario d'Une Histoire d'amour et de ténèbres d'après le roman d'Amos Oz. Il est aussi chanteur et compositeur de chansons, faisant partie du groupe « La bouche et les sabots ». C'est donc une personnalité très riche qui aime explorer de nombreux domaines.

Les Innocents est son cinquième roman et sa septième œuvre ; il a passé cinq ans à y travailler. Un seul autre de ses romans a été traduit en français : Crock attack (Tel-Aviv, 2006) qui a reçu le prix Courrier international. Il y alternait les chapitres ayant pour locuteurs un Israélien échappant aux attentats terroristes et un jeune Palestinien apparenté au réseau terroriste.

Ses autres oeuvres sont :

- Ice, Tel-Aviv, Gvanim, 1997

- Min babayit ha'almin (Sexe au cimetière), nouvelles, Tel-Aviv, zmora-bitan, 2000

- Moving (Déplacement), Tel Aviv, Zmora-Bitan, 2003

- Hydromania, Tel-Aviv, Zmora-Bitan, 2009

- Ukhal ba'amidah (Manger debout), Jérusalem, Ouganda, 2009

Récemment, il a publié avec son ami Edgar Keret Tel-Aviv noir, des nouvelles, Kinneret Zamora-Pavilion, 2014. 
Les Innocents a été traduit dans une dizaine de langues. Accueilli en Israël comme un grand roman national, car il est une des premières fictions à traiter d'un problème aussi épineux, il est un best seller en Israël et il y a été couronné par le Prix Bernstein. Mais il a reçu en France un accueil assez froid à cause du choix de l'auteur de ne pas diaboliser les implantés (qu'on appelle «colons » en France). Quoique de sensibilité politique différente et apparemment peu religieux, afin de connaitre de l'intérieur le mode de vie et la sensibilité des habitants, il a mené, pour rédiger son roman, une longue enquête, se rendant pendant cinq ans chaque week-end dans l'implantation de Tekoa Dalet, près de Gush Etzion, à une dizaine de kilomètres au sud de Bethléem, au pied de 1'Hérodion ; une implantation créée en 2001, après le début de la seconde Intifada, et peuplée de juifs à kippa tricotée. Modérée par rapport à certaines implantations de Samarie, celle-ci est pourtant considérée comme un avant-poste illégal et a été détruite par l'administration israélienne en mai 2015, sur une décision du ministre de la Défense, comme l'implantation du roman.

Puis, pour prendre de la distance, Assaf Gavron a rédigé la plus grande partie du roman à Berlin. Plein d'ironie et d'humour, son livre ne désire que rendre la lecture passionnante. Il se défend d'avoir voulu faire une œuvre polémique ou satirique, ni à l'encontre de l'administration israélienne, ni à l'encontre des implantés. Pour lui, la fiction a pour fonction de montrer la profondeur et la complexité des situations et des caractères, d'entrer dans la tête de personnages totalement différents afin de comprendre leur personnalité et d'être en empathie avec eux.

\section{Les Innocents}

Le roman se déroule sur une durée de cinq ans. Plus exactement, on suit la vie des implantés pendant un an car après le Prologue, qui évoque le choix du lieu et l'installation d'Othniel Assis, la première partie du roman contient dans son titre la mention: "Quatre ans plus tard». Quatre ans plus tard en effet, l'exploitation agricole initiale, plus ou moins tolérée, est devenue un îlot de peuplement (qui n'abrite cependant qu'une quinzaine d'habitants). C'est donc la vie de ces habitants qui est racontée, avec leurs tourments, les problèmes d'alimentation en électricité, et surtout les démêlés avec l'administration israélienne et la résistance aux tentatives d'expulsion. Le récit est centré, outre sur le responsable de l'implantation, Othniel Assis, sur deux frères, Gaby Nehoustan et Rony Cooper, qui représentent deux types d'Israéliens bien 
contrastés : Gaby, le religieux, disciple de Rabbi Nahman de Bratslav, solitaire et contemplatif; Rony, l'Israélien moderne et agnostique, toujours prêt à se lancer dans des entreprises hasardeuses, incapable de comprendre son frère.

Le roman présente une structure complexe, car la linéarité narrative est interrompue à plusieurs reprises par des analepses concernant le passé des frères. Le schéma de cette construction doit sans doute son inspiration au roman américain, Assaf Gavron ne cachant pas cette influence, en particulier celle de Jonathan Franzen qu'il considère comme un maître en écriture romanesque, avec Freedom ${ }^{2}$.

La structure du roman est la suivante :

- Prologue: installation d'Othniel Assis sur une colline près du Jourdain, à proximité du village arabe de Harmish.

- Première partie : «Les trois qui arrivèrent à la mi-journée. Quatre ans plus tard». Nous assistons à des visites d'officiels américains venus inaugurer une aire de jeux qu'ils ont financée et à une manifestation de gauchistes contre l'implantation, cependant que Rony, de retour des États-Unis, débarque chez son frère, et que l'épisode se termine par la célébration du shabbat.

- Deuxième partie: "Coupure d'électricité cérébrale». Première analepse : retour sur l'enfance tourmentée de Gaby, enfant solitaire et souffre-douleur, sur la mort accidentelle de ses parents, la vie au kibboutz, sa fugue et ses mésaventures à l'armée, tandis que son frère Rony réussit tout.

- Troisième partie : «Chaudes journées ». Retour à l'implantation où nous assistons à des transactions entre Rony et un paysan arabe, Moussa, pour commercialiser de l'huile «bio » dans les magasins de Tel-Aviv; à une naissance, à la construction par Gaby de son tzimer (cabane), à la vie quotidienne dans l'implantation, à la découverte de pièces anciennes dans une grotte, à la tentative par l'armée d'installer une ligne de démarcation, au scandale fait par la publication d'un article américain dénonçant l'implantation, à l'évasion d'un adolescent dans le monde virtuel d'internet, à la transaction sur l'huile faite par Moussa avec des Japonais (et non avec Rony)... et surtout, à la décision ferme du ministre de la

\footnotetext{
2 Jonathan Franzen, Freedom, Paris, éd. de l'Olivier, 2011, énorme roman mettant en scène des personnages assez déjantés de la classe moyenne, et nous faisant circuler dans un labyrinthe d'analepses qui rompent la continuité narrative mais qui permettent de sonder toute l'histoire des personnages et toute la complexité de leur psychologie.
} 
Défense d'évacuer l'implantation, scène restituée avec le plus grand comique.

- Quatrième partie : «Se nourrir de charognes ». Deuxième analepse, sur les traces des frères partis successivement aux États-Unis : Gaby comme dérivatif, où il travaille dans une entreprise qui récolte des fonds auprès de riches Américains pour les envoyer en Israël, et où il se marie ; Rony pour se lancer dans une carrière de trader qui se terminera très mal pour lui, à cause de placements imprudents, raison pour laquelle il vient se réfugier dans l'implantation. Échec total du mariage de Gaby et de l'éducation de son fils dont il se chargeait; sa rencontre avec des religieux qui le réconfortent et le convertissent décide de sa future orientation.

- Cinquième partie : «Retour à la base ». Alors que l'adolescent prend conscience de son erreur à propos du monde virtuel dans lequel il s'est réfugié, la vie dans l'implantation se complique avec des suspicions sur un indicateur, tandis que Yoni, le soldat éthiopien, tente de séduire la jolie jeune fille d'Othniel et que Nir Rivlin, pourtant marié, en rêve également. Une fois de plus, le générateur électrique tombe en panne, mais alors qu'on vient leur installer une alimentation électrique permanente (décision du ministère de l'Équipement) à l'occasion de Pourim, les troupes se mettent en place pour faire évacuer l'implantation alors que tous ses habitants sont en fête, déguisés, chantant, dansant. Suite à une ruse imaginée par Othniel, une espèce d'émeute mettant en scène les implantés confrontés aux Arabes du village voisin sur fond farcesque de Pourim, l'armée renonce.

- Le dernier chapitre célèbre avec lyrisme la permanence de la vie sur la colline.

Il s'agit donc d'un roman foisonnant qui brasse à la fois le quotidien de juifs religieux d'origines diverses (ashkénazes, marranes, sabras), isolés dans les collines où ils ont fait le choix de vivre, et leurs démêlés avec les autorités dont le manque de coordination permet à l'implantation de persister, en dépit des pressions internationales et du rôle des journalistes. Il s'agit aussi des relations avec les Arabes voisins, du contraste avec la vie à Tel-Aviv, des tentatives de réussir aux États-Unis, mais aussi des expériences sentimentales des jeunes ou moins jeunes. Nous pourrions aborder l'étude de ce texte à travers de nombreux aspects de la vie israélienne, sans compter avec la variété de son style, passant du descriptif au narratif insérant des dialogues, passant du lyrisme au comique, du pathétique à l'ironie, du grandiose au mesquin. 
Cependant nous avons choisi de nous centrer sur l'articulation particulière entre la fascination pour la nature et l'esprit religieux des protagonistes.

\section{La colline, la nature et la Bible}

\section{La colline, objet de fascination pour tous}

Dans de nombreux chapitres consacrés à l'implantation, le narrateur évoque la nature qui l'environne. C'est un lieu qui frappe par sa beauté, c'est donc un cadre magnifique que l'on tient à préserver et à ménager ; mais c'est surtout un personnage du roman, et les nombreuses personnifications intensifient sa forte présence pour les habitants.

Dès le début du roman, nous voyons le héros parcourir les collines et choisir le lieu où il s'installera :

Il traversa des rivières, visita des grottes, grimpa au sommet des collines avoisinantes, puis déboucha sur un terrain vaste et plat, qui n'était ni encombré par les oliviers de Harmish, le village d'à côté, ni trop rocheux. «C'est ici, dit-il, que je délimiterai mes champs ». $(12,15)^{3}$

Un choix qui s'impose à lui : une terre vierge, qui alimente tous ses rêves de vie naturelle, authentique, pionnière car « il aimait cette colline et ces vents et ce paysage antique » $(14,17)$.

C'est le même sentiment qu'éprouve Gaby lorsqu'il décide d'édifier son tzimer légèrement à l'écart :

Il se sentait chanceux. Il n'était jamais rassasié du paysage, des collines marron transparentes et des monts d'Edom. L'endroit à vrai dire était à couper le souffle. $(181,126)$

Ce sentiment est partagé non seulement par les habitants de l'implantation : « La nature est superbe ici, dit Nahoum en contemplant la nuit noire » $(246,168)$, mais aussi par les visiteurs, et même les forces de l'ordre qui tentent de faire appliquer les décisions d'évacuation :

Avec tout le respect que j'ai pour ce beau paysage, répondit le général de brigade, nous parlons d'un point stratégique. $(251,172)$

\footnotetext{
${ }^{3}$ Les chiffres qui suivent les citations renvoient, pour le premier, aux pages de l'édition en français et, pour le second, aux pages de l'édition en hébreu.
} 
La beauté du paysage exerce une sorte de fascination, elle impose le respect et le désir instinctif de la respecter, de la préserver. C'est ce que fait Gaby lorsqu'il construit son tzimer :

Gaby fit de son mieux pour harmoniser sa cabane au paysage fantastique, pour abonder dans son sens, et ne pas le blesser. $(182,126)$

C'est pourquoi, l'intrusion de l'homme au sein de ce paysage grandiose est perçu comme une agression, une blessure :

Colline. La terre est claire, silencieuse, presque vide : un jaune-marron lardé de rochers, d'oliviers solitaires, et de taches qui forment une couverture d'un vert tendre d'après la pluie. Une route à sens unique, étroite et cabossée, éventrait le centre de la colline. $(25,25)$

Déjà, derrière l'opposition entre la douce « couverture vert tendre » de l'herbe qui semble la préserver comme un enfant et la route qui l'éventre, nous repérons une personnification qui revient à de nombreuses reprises au fil du roman, les collines étant vues comme une femme pudique car elles « se drapaient du voile verdâtre de l'oseille, à la plus grande joie des chèvres et des moutons, toutes nationalités confondues » (189, 131). C'est une nature généreuse puisqu'elle nourrit, comme le suggère avec humour le narrateur, le bétail tant israélien que palestinien, cela semblant lui être bien indifférent, une nature bienveillante envers les animaux, une nature habitée puisqu'à la fraîcheur retrouvée de la nuit on pouvait en effet « entendre les grenouilles coasser, les stridulations des sauterelles, le sifflement des grillons...» $(51,41)$.

On appréciera ici le rythme ternaire qui renforce cette impression de perfection de l'environnement dont bénéficient les habitants de l'implantation, son caractère vivant, « habité », et l'on conçoit que les implantés se sentent tout à fait à l'unisson avec la Nature.

Cette impression de vie est renforcée par les personnifications utilisées par l'auteur. La nature a bien une vie propre qui semble se communiquer aux habitants, à moins que ce ne soit un fait de description symbolique et l'effet d'une projection de l'état des humains, assommés par l'intensité du soleil, sur la nature :

Un soleil chauffé à blanc surplombait les montagnes somnambules. $(189,131)$

Il en va de même pendant l'hiver : 
Le froid se posa sur la colline pendant la nuit et, au matin, le gel scintillait entre les mottes de terre, sur les outils, les cactus [...] Le jour ouvrit les yeux sur un large bâillement, et de très longues heures s'écoulèrent avant qu'il ne se défasse du froid. $(619,409)$

Ce mélange qui fait alterner les éléments naturels et le bric-à-brac des habitants serait-il le reflet d'une symbiose particulièrement réussie entre les habitants et le paysage ? Peut-être, mais sans doute aussi l'auteur tient-il à montrer que le paysage, que la colline est un véritable personnage dans le roman, et c'est certainement pour cela qu'il avait donné ce titre La Colline à son œuvre. Il va plus loin encore en prêtant aux éléments une personnalité joueuse et indépendante, qui refuse de se plier à la volonté des hommes :

En hiver, il y a de si belles journées que même les nuits les plus froides s'adoucissent, et qu'on en oublie la rudesse. Un soleil effronté, railleur, souriait au dessus de la colline, la météo annonçait un proche refroidissement, mais le soleil s'en fichait complètement et la chaleur ne cessait d'augmenter. $(610,404)$

Non seulement la nature trompe les prévisions, mais elle impose sa loi aux hommes. Juste après la tentative d'expulsion des habitants par l'armée, mise en échec par une bataille rangée entre les habitants de l'implantation et les Arabes du village voisin, la nature impose le calme, le retour à la paix, au silence :

La neige persista à tomber trois longues journées. Elle recouvrit la région, imposa le silence; les collines clignaient de toute leur blancheur; en contrebas, les paysages lointains, désertiques, fusionnaient en un beige plus clair que d'habitude, et se réfléchissaient dans le ciel blanchissant qui aveuglait le soleil, apparu timidement tête basse. $(641,424)$

Ainsi, l'auteur a éprouvé par ses sens, lors de ses séjours au fil des saisons dans une implantation, le caractère de cette nature fascinante et qui inspire le respect, et il restitue sa perception par une écriture impressionniste qui nous aide à nous identifier aux habitants plongés au sein des collines de Maaleh Hermesh, où par sa proximité la nature impose sa présence, sa loi, sa réalité qu'on a tendance à oublier dans la vie urbaine.

Cependant, il est curieux que la nature soit vue uniquement comme merveilleuse, en quelque sorte par l'œil d'un touriste, et jamais du point de vue de la difficulté du travail qu'elle donne, et pourtant, ses habitants cultivent la terre, cette terre désertique, aride, où ne poussent que des 
oliviers, là où il fait trop chaud l'été et glacial l'hiver. Mais apparemment, cela ne compte pas. La terre, en effet, semble avoir pour les implantés une signification symbolique beaucoup plus importante que le mal qu'elle peut leur imposer : elle est toujours ici généreuse, belle, " olympienne » en quelque sorte, et jamais ingrate, dure ou rebelle. C'est pourquoi elle est souvent associée à des évocations bibliques.

\section{La nature associée à la Bible dans le roman}

Dès l'ouverture du roman, le décor est planté, le lecteur est mis en condition: il ne s'agit pas seulement d'une colline, mais plutôt d'un commencement, comme si le narrateur nous invitait au tout début de la Création, à la Genèse. D'emblée il est placé en focalisation interne, et c'est bien de cela qu'il s'agit pour le fondateur lorsqu'il choisit cette implantation. Le lecteur français est frappé par la résonance biblique de l'incipit, qui ne se trouve pas vraiment dans le texte hébreu où l'on s'attendait à trouver bereshit. L'auteur, conscient de l'analogie biblique, déclare à ce sujet qu'il a délibérément choisi de moderniser le texte :

$\mathrm{Au}$ commencement, il y eut les champs. En ce temps-là, Othniel Assis était installé à Maaleh Hermesh, où il élevait une chèvre pour son plaisir, tout en cultivant de la roquette et des tomates cerises dans le jardin de sa maison. La chèvre était destinée aux enfants; la roquette et les tomates, aux salades de son épouse Rachel. Othniel vit que tout cela était très bien. $(11,15)$

Mais visiblement, ce ne sont pas seulement les personnages qui projettent leur vision du monde sur le paysage. La terre elle-même «crie » pour manifester cette virginité : un sol farouche que Dieu luimême a formé et qui garde encore son empreinte. Elle crie, mais aussi murmure et joue de la musique: le triple rythme ternaire lui-même semble restituer la perfection initiale :

Et par delà, omniprésent - le paysage royal, sublime et sauvage, qui paraissait crier, parfois murmurer, ou même jouer : c'est ici que se trouve le désert. Là est le Livre. Là est la Genèse.

«Quel air!», dit Sheldon Mamelstein, puis il respira à pleins poumons. Dans la lumière d'après le crépuscule, le paysage semblait lunaire. De là ils pouvaient se représenter la Création, comme si le cosmos lui-même avait été formé ainsi, avant de se stabiliser. $(35,31)$

La sage-femme qui parcourt les collines pour aller aider une jeune femme à accoucher à Maaleh Hermesh 3 fait le même constat : 
Un paysage digne des jours de la Genèse. $(214,147)$

Aussi n'est-on pas étonné de constater que les régions environnantes sont nommées par leur nom biblique. Ainsi, pour l'est du Jourdain :

Le soleil s'éleva au dessus d'Edom et de Moab, un jour nouveau enveloppa la colline, et le pays s'emplit d'une lumière dorée. $(216,149)$

Tout est bon pour citer la Bible, lorsque devant un paysage, ces paroles montent naturellement aux lèvres des implantés : ici, au moment d'entrer dans une grotte chargée d'histoire :

Néhama cita les paroles du prophète hébreu Amos, qui avait décrit ce paysage « les montagnes ruisselleront de moût et les collines se liquéfieront ». $(294,198)$

Pour ces personnages, la terre semble être le lien indispensable qui leur permet de vivre authentiquement leur vie religieuse, comme s'ils devaient absolument vivre comme les personnages de l'Ancien Testament, une vie d'éleveur, d'agriculteur, et que ce sont les seuls modèles qu'ils reconnaissent. La vie urbaine constituerait pour eux une rupture radicale avec leur idéal de vie :

Le berger ne s'attache à aucun endroit, il quitte un lieu sûr, familier, pour atteindre un objectif encore plus haut, qui est de s'unir au Créateur et à la vie de l'esprit. Il contemple le monde et élargit ses horizons intérieurs, tandis que le paysan est assujetti à ses possessions [...] L'agriculteur a une base solide et régulière. Il crée quelque chose - il ensemence, il en tire les fruits, et ne fait pas que s'asseoir à l'ombre, pour laisser son troupeau faire le boulot. Notre peuple, depuis les jours de sa genèse, a vécu sur ces contrastes : Caïn et Abel, Avraham et Isaac, même rabbi Éléazar débuta comme agriculteur, tandis que rabbi Akiva était berger. Quant à Othniel Assis, il est les deux à la fois. $(180,125)$

Choisie et habitée comme un lieu biblique par excellence, la colline semble frapper chacun par son caractère premier, essentiel. Lorsqu'on parcourt des montagnes ou des collines loin de toute habitation humaine, de toute trace humaine (cabane, câble téléphonique etc.), on ressent précisément cette impression puissante d'être transporté au quatrième ou cinquième jour de la Genèse, lorsque la nature a pris forme et qu'elle ne semble habitée que par la présence animale et végétale. C'est aux mêmes exemples bibliques que pense Gaby lorsqu'il parcourt la colline en 
compagnie de ses chèvres, « comme Abel, comme Abraham, notre Père, comme David, notre Roi, comme Moïse notre Maître » $(649,429)$.

Et quoi qu'il se passe dans la nature, tout ramène les habitants à leur Dieu, à sa puissance. C'est le cas lorsqu'une tempête s'abat sur la région, et à ce moment-là, toutes les créatures, même les oliviers, semblent redouter cette colère d'une nature associée à Dieu :

Un jaune apocalyptique dans les cieux, des vents si forts que même les plus vieux troncs d'oliviers semblaient renoncer, ployaient, et une pluie torrentielle qui balayait l'ensemble [...] à la réflexion et au respect pour la force sans concession de la nature, de Dieu, tout cela mêlé à un léger sentiment de crainte face à une telle puissance. $(517,345)$

\section{Descriptions de la nature dans le style biblique}

Mais l'auteur ne se contente pas de mentionner explicitement la Bible lorsqu'il évoque la nature ; pour mieux faire goûter cette ambiance particulière, il écrit certains passages dans le style biblique, pastichant la poésie du Livre :

Les vents passeront, les jours deviendront orphelins, mais la vie continuera : les enfants grandiront, les fidèles prieront, les oliviers romains, dans leur écrasante majorité survivront comme ils l'ont fait pendant des milliers d'années, [...] des gens qui furent et qui ne sont plus, ou qui ont pris leur propre voie, à moins qu'ils n'aient déjà achevé le rôle qu'ils devaient jouer. Un Dieu unique, saint et majestueux, qui voit et qui sait tout. Une petite colline au milieu de nulle part, au milieu de partout, avec quelques rochers, des ronces et des âmes. (649-650, 429)

Il est difficile pour le lecteur de ne pas penser ici au livre de l'Écclésiaste, à la fois à son style mais aussi à sa philosophie, l'idée qu'il $\mathrm{y}$ a un temps pour tout, et que tout est vain, comme à la fin du roman, lorsqu'une fois de plus l'armée a renoncé à évacuer l'implantation, et que la vie continuera, et surtout que la colline continuera, superbement indifférente à ce qui arrive autour d'elle, une « colline au milieu de nulle part, au milieu de partout ». Comment mieux signifier que les drames vécus ne sont que contingences temporelles qui n'affectent en rien la marche du monde ? Et c'est sur les deux dernières phrases du passage que s'achève le roman, associant Dieu et la colline, tous deux éternels et loin de la zizanie des hommes.

Ailleurs, l'auteur joue de la répétition afin de marquer l'importance de ce moment où le temps s'arrête, moment de contemplation où il est donné de goûter l'instant, le moment du shabbat : 
Le shabbat descendit sur la colline comme une coiffe sur des cheveux, doux et plaisant $[\ldots](69,52)$

Le shabbat descendit sur la colline comme un voile sur les épaules d'une mariée, silencieux, éthéré $[\ldots](69,52)$

Le shabbat descendit comme un générateur qui tombe en panne [...]

Le shabbat descendit comme un soleil déclinant, accompagné de vents paisibles. $(70,52)$

Le shabbat descendit sur la colline comme la pluie, fraîche et abondante. $(74,55)$

Le shabbat se posa sur Maaleh Hermesh 3 telle une navette spatiale sur la lune, de façon précise et déterminée. $(218,150)$

La répétition qui rythme de manière poétique certaines pages parfois fort éloignées les unes des autres fait penser au texte biblique, d'autant plus qu'il y est question du shabbat. En effet, la répétition est un procédé assez fréquent de la poétique biblique. Ce peut être une simple répétition de mot, mais aussi quelquefois de syntagmes complets, comme par exemple dans le livre d'Isaïe :

Éveille-toi, éveille-toi ; revêts-toi de force,

bras du Seigneur (Is. 51,9)

Réveille-toi, réveille-toi,

mets-toi sur pied, Jérusalem (Is. 51,17)

Éveille-toi, éveille-toi,

vêts-toi de ta force, Sion (Is. 52,1)

Ou encore les répétitions que l'on trouve dans le livre de la Genèse de « Dieu dit... », de « Dieu vit que cela était bon», ou de «Il y eut un soir, il y eut un matin ».

Cet effet rythmique rend le texte particulièrement poétique. De plus, en nous plongeant ainsi dans le rythme vécu par les habitants, il donne à goûter l'atmosphère particulièrement religieuse qui règne dans l'implantation, et c'est comme si le cosmos entrait en shabbat et poussait les hommes à y entrer eux-mêmes. Toutefois, les images très prosaïques peuvent surprendre (celle du générateur dont la panne doit être fort embarrassante au moment du shabbat, puisqu'il est impossible d'aller le relancer, ou celle de la navette spatiale), provoquant un effet de rupture cocasse au milieu de l'atmosphère irénique et recueillie.

Ainsi, visiblement l'auteur s'amuse : à la fois il restitue le vécu des habitants, des gens très religieux, mais avec des ruptures et des décalages, il oblige à prendre de la distance, comme à ne pas nous laisser 
prendre dans sa fiction. Il demeure le maître du jeu, sans toutefois sortir de son regard empathique vis à vis de ces juifs très religieux.

\section{La pratique religieuse des habitants de Maaleh Hermesh 3}

\section{Attachement au texte biblique et aux traditions}

Les habitants de l'implantation sont tous des juifs religieux mais toute leur vie est scandée, marquée par les rappels des événements bibliques. C'est ainsi que le calendrier est inévitablement noté par les mois bibliques, qu'il s'agisse d'Av, d'Adar ou de Tammouz :

Le mois de Tammouz venait de s'achever, et on entrait dans la période qui marque le deuil de la destruction de Jérusalem par les légions romaines [...] C'est le soir du shabbat de la vision qui est le shabbat précédant le neuvième jour d'av - [jour de la destruction de notre Sanctuaire, à Jérusalem, par les armées romaines, pour cause de haine gratuite qui sévissait alors parmi le peuple d'Israël]. ${ }^{4}(304,205)$

Ici le narrateur pousse la focalisation interne jusqu'à utiliser une énonciation à la première personne du pluriel, selon l'usage des juifs pieux, mais n'éprouve pas le besoin d'expliciter l'événement historique, contrairement au traducteur en français qui suppose un lecteur ignorant de cette histoire d'Israël.

Mais toutes les occasions de la vie sont propres à faire surgir un événement biblique ou un verset. Ainsi, c'est le cas de Nir Rivlin, qui est en assez mauvais termes avec son épouse, mais est très attiré par la jeune Guittit Assis qui, à la nuit tombée, va rejoindre le soldat éthiopien Yoni. Prenant conscience de son attirance, il s'admoneste et :

Il se souvenait aussi [du récit biblique] de Joseph, qui avait su maîtriser son désir [pour l'épouse égyptienne de Putiphar]. $(336,224)$

Notons à nouveau la nécessité ressentie par le traducteur de préciser de quel événement biblique il s'agit, alors que le texte hébraïque, faisant appel à un présupposé connu, est spécialement concis. Visiblement, la Bible constitue pour les implantés un code moral qui les aide à adopter une conduite honorable. Elle sert également d'argument au

\footnotetext{
${ }^{4}$ Les passages entre crochets sont des ajouts du traducteur du roman et les mots en italiques sont dans le texte.
} 
jeune soldat Jacobi pour tenter de convaincre son supérieur de le laisser emmener le chien qu'il vient de trouver :

Jacobi invoqua la Tora et l'impératif de bonté envers les animaux. $(314,211)$

La Bible leur sert aussi d'argument pour défendre leur droit à demeurer là où ils sont : " Cette terre est à nous, comme dit la Bible sans votre bullshit de me dire quoi faire !» $(552,369)$, déclare Josh, le marrane ayant retrouvé ses racines, à l'officier venu les expulser. Un peu auparavant, avant de décider de la bonne manière de résister au décret d'expulsion, ils s'admonestent en se sentant encouragés par la tradition biblique : "Il exerce sa vindicte sur ses ennemis, réhabilite sa terre, et son peuple » $(568,378)$.

Mais le Talmud aussi les habite et est invoqué lorsque c'est nécessaire. Othniel, le chef de l'implantation, se sert de ce passage tiré de Sanhédrin, 19-9, pour démasquer l'indicatrice qui fournissait des renseignements aux autorités et la manœuvre fonctionne immédiatement, Jenya Freud éclate en sanglots et passe aux aveux rapidement devant cette mise en accusation :

Ne prends garde ni aux pharisiens, ni aux non-pharisiens, ni aux sadducéens, ni aux non-sadducéens, mais prends garde aux hypocrites qui accomplissent l'acte de Zimra et en exigent la rétribution de Pinchas. (360-361, 241)

Mais avant tout, la Bible qui leur monte aux lèvres à tout propos est réconfort dans l'épreuve. Et c'est tout naturellement que Gaby invoque le Psaume 23, en particulier lorsqu'il se trouve dans des moments de grande difficulté :

Même si j'allais dans la vallée de l'ombremort, je ne craindrais rien car tu serais à mes côtés. (492 et 541,327 et 361 )

Dieu est mon berger, je ne manquerai de rien. $(576,383)$

Mais les habitants ne se contentent pas d'invoquer Dieu et la Bible dans les moments de tension ou de désespoir, ce qui, somme toute, est très humain. Leur quotidien est tissé de Bible dont les paroles accompagnent chacun de leurs gestes. Ainsi Othniel, lorsqu'il s'apprête à recueillir le chien, abandonné à grand regret par Jacobi, ne peut s'empêcher d'accompagner son geste d'une bénédiction : 
Celui qui aide une seule créature en Israël, c'est comme s'il subvenait aux besoins du monde entier. Dieu a donné, Dieu a repris. Que son nom soit sanctifié. (316, 213)

Les citations bibliques qui montent spontanément aux lèvres des personnages sont toujours rigoureusement fidèles au texte, si ce n'est qu'ici le terme hashem remplace le tétragramme, imprononçable. Ainsi les femmes et les enfants, lors de leur pique-nique, ne manquent pas d'accompagner chacun de leurs gestes d'une bénédiction :

Ils sortirent sandwichs, boissons ; ils se lavèrent les mains et, avant de mordre dans les sandwichs, récitèrent la bénédiction appropriée: «Béni sois-Tu, Éternel, Roi de l'Univers, qui fais surgir le pain de la terre ». $(293,197)$

Ici l'auteur israélien prend bien soin de donner l'ordre précis du rituel, afin de bien marquer à quel type d'observants nous avons affaire, alors que le traducteur trouve cela sans doute trop complexe à exposer à un public profane et simplifie l'action.

Hommes, femmes et enfants baignent dans cette ambiance pieuse : deux petites filles, pendant ce même pique-nique, chantent un extrait du Cantique : "C'est la voix de mon bien-aimé, et le voici qui vient» (293, 197). Ailleurs, une jeune femme et ses filles chantent un autre passage biblique : «Elles passèrent au cantique qui loue les vertus de la femme vaillante $»(578,384)$.

Bien sûr, Rony le mécréant porte un regard très critique sur les habitants de l'implantation (qu'il finit par quitter) où son frère et ses amis :

[...] philosophaient sur les figures bibliques de Jacob, Joseph, Esaü et sur le Saint, béni soit-Il. Comme ils aimaient deviser! Combien de commentaires, d'exégèses, sur des histoires bibliques ! ( 524,349$)$

Ainsi l'implantation vit au même diapason, dans la même ambiance harmonieuse, avec les mêmes références, et Jéhu, sur son cheval, peut vérifier que tout est tranquille et que chacun est bien " sous sa vigne et sous son figuier $»(298,201)$ (guillemets et note du trad. : I R. 5,5).

L'onomastique est un autre point qui peut paraître insignifiant car il est relativement habituel en Israël. De fait, les personnages principaux du roman portent des noms symboliques. Et tout d'abord, le chef de l'implantation: Othniel, sur lequel s'ouvre le roman. Ce n'est sans doute pas par hasard si l'auteur a choisi ce prénom pour celui qui, à force de 
relations, de résistances, de vigilance et de ruses, arrive à préserver la communauté de toutes les attaques et de toutes les tentatives de les déloger. En effet, Othniel est le nom d'un héros biblique qui a sauvé Israël dans ses débuts ( Jos.15,17; Jg 1,13 ; 3,7-11 ; 1 Ch 4,1-13).

De même, l'autre héros du récit, se nomme Gaby Cooper, et se fait appeler maintenant Gabriel. Pourquoi donc lui avoir choisi le nom d'un archange (Dn.8,16;9,21), alors que c'est précisément celui qui, dans le roman, passe pour Le saint homme ? De plus, il a hébraïsé son nom en Nehoustan, traduction du cuivre que signifie le patronyme Cooper, et l'auteur lui fait dire, non sans un grain d'humour et pour se défendre des railleries de son frère, qu'il s'est appuyé pour ce faire sur une autorité incontestable :

Un rabbin, un rabbin érudit et spécialiste des patronymes juifs, m'a dit que vraisemblablement ce nom est lié au cuivre. $(308,208)$

Tous, dans ce milieu, aiment se trouver un prénom puisant sa source dans la Bible, ainsi la sage-femme qui vient accoucher une jeune femme à Maaleh Hermesh 3 :

Elle avait pris le nom de Shifra, qui est celui de la sage-femme biblique de l'Exode. D'elle, comme de son amie Pua, il est écrit : "Mais les sages-femmes craignaient Dieu » ( (Ex, 15-19) - or elle aussi Le craignait, et Lui, que son nom soit béni, la protégeait. $(214,147)$

Alors, lorsqu'un enfant naît, il faut lui trouver un prénom significatif qui enchantera les parents :

Yemima, comme la merveilleuse fille de Job, ou encore comme l'expression yamim-yemima qui exprime le lien historique, donc l'enracinement dans les générations antiques. $(328,219)$ (Job 42,14)

Ainsi tout montre la piété des habitants de Maleh Hermesh 3 : prière à la synagogue, moment du shabbat sacralisé, citations et exemples bibliques sans cesse sur les lèvres, tenue vestimentaire, comportement ; cette implantation, pour petite qu'elle soit (en tout dix-sept personnes avec les enfants !) est vraiment faite d'hommes et de femmes religieux dont la relation particulière à la nature, le lyrisme du regard porté sur le paysage s'explique par une piété particulière, qui se rattache au hassidisme, et plus particulièrement au Rabbi Nahman de Bratslav. Or il y a dans cette piété, qui accorde une si grande importance à la Nature, 
quelque chose de mystique, loin de la simple orthopraxie des juifs même très observants. Du reste, ce type d'attitude n'est pas propre au judaïsme ; il rejoint une spiritualité que l'on peut trouver, par exemple, dans le christianisme chez un François d'Assise. Et visiblement, cette spiritualité joue un rôle fondamental dans l'installation et le maintien de l'implantation sur cette colline.

\section{La nature dans la Bible et la tradition}

Aujourd'hui, pour une grande partie des croyants, la relation entre religion et nature ne semble pas évidente. Stanislas Breton, éminent philosophe catholique, soutient que le terme «nature» ne fait pas partie du langage religieux ${ }^{5}$. Par ailleurs, en Occident au moins, la relation Dieu-Homme a été mise en avant dans les théologies chrétiennes, et ce n'est que récemment que s'est éveillée, avec la montée des mouvements écologiques, une sensibilité à une «écologie biblique » et la prise de conscience que Dieu a fait de l'homme le partenaire de la Création et non son simple utilisateur, maître et destructeur comme la modernité a eu tendance à l'induire.

Dans le judaïsme, il semble qu'il en soit de même. Paul Celan déclare que « le juif et la nature, cela fait deux, de tout temps, et même aujourd'hui, même ici $»^{6}$. Catherine Chalier impute leur distance vis à vis de la nature (dont ils craignaient la divinisation possible) aux conditions de vie imposées aux juifs dans le passé et qui les écartaient du travail de la terre. Pourtant, la Bible mentionne souvent la nature, en tant que fruit de la Création de l'Éternel, et les Hébreux, en tant que ruraux en contact permanent avec la nature. La Genèse, en effet, parle du ciel, de la terre, des mers, des herbes, des arbres, des oiseaux etc, créatures, tout comme l'homme. Et chaque création est ponctuée de l'approbation de Dieu : «Dieu constata que c'était une bonne chose». (Gn I, 4.10.12.18.21.25.31), et finalement il le constate pour toutes ses créatures sauf... pour l'homme!

Noé, quant à lui, sauve le monde vivant (Gn 7-8) en faisant monter dans son arche un spécimen de chaque espèce et la colombe qu'il envoie à la fin du Déluge rapporte une feuille d'olivier. Il est le seul «juste»

\footnotetext{
${ }^{5}$ Stanislas Breton, « Christianisme et concept de nature », dans Dominique Bourg (dir.), Les Sentiments de la nature, Paris, La Découverte, Paris, 1993, p. 146.

6 Paul Celan, «Entretien dans la montagne », trad. John E. Jackson et André du Bouchet, dans Strette, Paris, Mercure de France, 1971, cité par Catherine Chalier dans L'Alliance avec la nature, Paris, Cerf, 1989.
} 
sauvé avec sa famille alors que Dieu a décidé d'éliminer les hommes mauvais. Néanmoins, la Création, même s'il reste un spécimen de chaque espèce, paiera pour la perversion de l'homme, et il n'est pas difficile ici d'en déduire la solidarité de la Création entière.

Et tout au long de la Bible, la nature est présente avec de nombreuses évocations de paysages ou de personnages occupés aux travaux des champs : Moïse gardant des moutons (Ex 3,1), Saül cultivant les champs (I S 11,5), David faisant paître les brebis (I S 17,34) ou encore Amos berger cultivant les sycomores (Am 7,14-15).

Il semble que la Création réfute la seule relation duelle Dieuhomme, mais que toujours la relation est triangulaire : lorsque Job est réduit à l'état de débris humain et qu'il réclame la justification de sa souffrance, Dieu lui répond par la description de la richesse de sa Création, comme s'il devait trouver en elle la justification demandée ( $\mathrm{Jb}$ $38)$ : « Où donc te trouvais-tu quand je fondais la terre ? » $(38,4)$, comme pour le ramener à l'humilité nécessaire à la compréhension de son œuvre. C'est à cette même humilité qu'est amené le psalmiste dans la contemplation des éléments :

Quand je vois tes cieux, œuvre de tes doigts, la lune et les étoiles que tu as fixées qu'est donc l'homme pour que tu penses à lui, l'être humain pour que tu t'en soucies ? (Ps. 8,4-5)

$\mathrm{Du}$ reste, la Création elle-même loue Dieu, et pas seulement l'homme, comme c'est le cas dans le Psaume 19 (2 et 5-6) :

Les cieux racontent la gloire de Dieu, le firmament proclame l'œuvre de ses mains. [...] Leur harmonie éclate sur toute la terre et leur langage jusqu'au bout du monde. Là-bas, Dieu a dressé une tente pour le soleil : c'est un jeune époux sortant de la chambre, un champion joyeux de prendre sa course.

ou le Psaume 65 (10 et 13-14) :

Tu as visité la terre, tu l'as abreuvée ; tu la combles de richesses. La rivière de Dieu regorge d'eau, tu prépares le froment des hommes. Voici comment tu prépares la terre [...] Les pacages du désert ruissellent, les collines prennent une ceinture de joie, les prés se parent de troupeaux; les plaines se drapent de blé : tout crie et chante.

Par ailleurs, la Bible n'est pas avare de personnifications décrivant les éléments naturels comme célébrant la gloire du Très-Haut : 
Que les cieux se réjouissent, que la terre exulte, et que grondent la mer et ses richesses! Que la campagne tout entière soit en fête, que tous les arbres des forêts crient alors de joie. (Ps 96,11-12)

Que grondent la mer et ses richesses, le monde et ses habitants! Que les fleuves battent des mains, qu'avec eux les montagnes crient de joie! (Ps 98,7-8)

Ici, le psalmiste ne distingue pas parmi « les habitants » du monde, et sans doute s'agit-il non seulement des hommes, mais encore de toutes les créatures. C'est pourquoi le livre de la Sagesse, deutérocanonique il est vrai, observe : «Car la grandeur et la beauté des créatures conduisent par analogie à contempler leur Créateur» $(13,5)$. Mais cette contemplation « par analogie », ne se rencontre-t-elle pas ailleurs dans le texte biblique ? Car dans l'Ancien Testament, il s'agit essentiellement de prières de demandes : demandes de protection, de guérison, de sagesse, de prospérité, de délivrance, qui ne sont pas mises en relation avec la nature. Quant aux prières de louange et de contemplation, on les trouve essentiellement dans les Psaumes:

J'étais ainsi quand je t'ai vu dans le sanctuaire en contemplant ta force et ta gloire. (Ps. 63,3)

J'ai demandé une chose au SEIGNEUR, et j'y tiens: habiter la maison du SEIGNEUR tous les jours de ma vie, pour contempler la beauté du SEIGNEUR et prendre soin de son temple. (Ps. 27,4)

Levez les mains vers le sanctuaire et bénissez le SEIGNEUR. (Ps. 134,2)

On le constate, la contemplation est ici liée au Temple, au Sanctuaire, et il ne saurait y avoir d'ambiguité : la «maison du Seigneur », c'est le Temple, et ce n'est pas la nature. Et de fait, le sentiment d'étrangeté que nous avons éprouvé au spectacle des personnages du roman tellement habitués à cette prière contemplative dans la nature est bien un phénomène particulier, obéissant à une spiritualité particulière.

Et dans la pratique, si les prières juives se réfèrent toutes au Dieu «Créateur » et prennent une dimension forcément cosmique, il n'y est pas fait de référence spécifique à ce que nous appelons «nature ». Cependant il faut mentionner que le terme bénédiction (berakha) est un mot dont la racine (BRKH) évoque la nature, car elle désigne une bouture. Ainsi, le lien intrinsèque entre Bible, judaïsme et nature est toujours sous-jacent dans les pratiques juives.

C'est le cas des fêtes, qui au fil de l'histoire ont vu leur signification évoluer, passant d'une signification «agraire» à une signification 
« historique », puisque se référant aux grands événements ayant marqué l'histoire du peuple hébreu. Il en va ainsi des trois grandes fêtes de pèlerinage (Pessah, Shavouot et Soukkot).

De plus, certaines traditions religieuses ramènent forcément le croyant à la nature : c'est en particulier celle du loulav, à Soukkot, qui consiste à rassembler quatre espèces de plantes : un cédrat, une palme de dattier, une branche de myrte et une branche de saule. Il est clair que de nombreuses interprétations rivalisent pour donner la signification de ce loulav, qui peut signifier que «le règne d'Hachem s'étend à toutes les extrémités de l'univers » (Talmud Soucca 37b). Mais il peut représenter aussi les différents types de piétés juives ou les différents types d'hommes.

Autrement dit, pour la piété juive, la nature n'est pas seulement un « recueil de biens matériels», mais surtout un « recueil de significations $»^{7}$. C'est pourquoi Marc-Alain Ouaknin ${ }^{8}$ parle non pas d'écologie, mais de véritable éco-logos, pour évoquer le lien du judaïsme avec la nature et le « discours » de l'univers.

Ainsi, il est indéniable que le judaïsme entretient des liens avec la nature, les éléments, le cosmos; il y puise ses racines et quasiment sa justification, même si cela demeure souvent implicite dans la pratique. Et c'est cette "essence du judaïsme» qu'ont sans doute retrouvée les hassidim du XVIII ${ }^{\mathrm{e}}$ siècle ainsi que leurs épigones d'autrefois et d'aujourd'hui, de par le monde et en Israël.

\section{L'articulation Bible-Nature dans le roman}

Ainsi, nous sommes fondés à nous interroger sur la piété particulière des habitants de l'implantation car, malgré le sentiment d'un panthéisme sous-jacent à leur attitude, il semble que leur spiritualité ne soit pas si hétérodoxe ni vraiment étrangère à la Bible elle-même.

Certes, le danger de panthéisme est toujours présent, donc celui de l'idolâtrie, et il est évidemment souligné dans la Bible elle-même. Et d'ailleurs, au nom de ce danger, les mitnagdim ont préféré s'en tenir à la stricte observance et faire la guerre aux hassidim illuminés qui privilégiaient la spontanéité. Certes, l'idole, «ce visible qui s'empare

\footnotetext{
${ }^{7}$ Introduction de Giuseppe Angelini au volume de A. Caprioli, L. Vaccaro, Questione ecologica e coscienza cristiana, Morcelliana, Brescia, 1988, cité par Massimo Cassani dans une conférence donnée à Luxembourg, le 7 novembre 2005.

${ }^{8}$ Marc- Alain Ouaknin, Tsimtsoum, Paris, Albin Michel, 1992.
} 
indûment de l'invisible, le limite et l'opacifie $)^{9}$, est un danger dont ont conscience les maîtres du hassidisme, et un danger qui guette toujours l'homme épris d'immédiateté, c'est pourquoi ils ont toujours prescrit l'étude au même titre que la prière intense. Ils réfutent à la fois l'étude seule qui dessèche et coupe du monde, et la contemplation seule qui risque de diviniser la nature : les deux sont inséparables à leurs yeux.

C'est pourquoi, dans le roman, Gaby ne va jamais contempler, méditer et prier sur les collines sans ses livres d'étude, les deux étant indissociables. C'est ce que désiraient R. Menahem de Prémishlan, le Baal Shem Tov, R. Schéour Zalman de Liadi et Nahman de Bratslav.

Autrement dit, ces maîtres avaient opté pour une piété s'appuyant autant sur la transcendance que sur l'immanence de Dieu, donc d'un Dieu qui n'est pas absent du monde, qui continue de veiller sur ses créatures. Et c'est ce que vivent intensément les personnages à l'heure du danger : ils ont confiance. Alors qu'ils fêtent Pourim, que le carnaval bat son plein tandis que les troupes, les chars et les hélicoptères s'amassent aux portes de l'implantation pour les chasser, ils gardent confiance :

De toute façon, même s'ils entreprennent quelque chose, Pourim est un jour de miracles, d'annulation des mauvais décrets. $(621,410)$

Et de fait, le roman leur donne raison puisque, conjointement à la ruse d'Othniel qui consistait à aller provoquer les voisins arabes pour susciter une émeute redoutée au plus haut point par les autorités, les éléments s'en mêlent pour décider que cela a assez duré :

Des pneus furent incendiés, et il en monta une fumée noire qui empuantit l'air froid [...]. Alors la neige cessa d'hésiter, et tomba pour de bon : en flocons épais, lents, moelleux, royaux. $(640,423)$

Comment mieux signifier que la Providence à laquelle croient les habitants de Maaleh Hermesh 3 veille sur eux, sinon par cette ironie de la personnification et par cette accumulation d'adjectifs exprimant la sérénité transcendante, opposée à l'excitation et à la panique des hommes.

Ainsi cette piété confiante, et un peu naïve pour le regard extérieur, finit par triompher : oui, la nature est de leur côté, elle parle le langage de Dieu, confirmant l'affirmation du Psalmiste : «la terre est remplie de la fidélité du SEIGNEUR ». $(33,5)$.

9 E. A. Levy-Valensi, Lettres de Jérusalem, Bruxelles, L. Musin, 1983, cité par Catherine Chalier, op. cit. 
Et l'on ne peut pas dire qu'il s'agisse d'une foi déjantée, hérétique ou déviante. La plupart des références qui font le lien entre la nature et la Bible proviennent du livre de la Genèse ou des Psaumes. Rien ici qui contrecarre le texte biblique, car il s'agit de la confiance au Dieu Créateur et au Dieu de l'Alliance et de sa célébration que la Tora leur enseigne sans que les personnages semblent mener une « existence déchirée entre la nature et la loi $»^{10}$. Même leur assurance et leur foi reposent sur l'Alliance donnée dans la Tora, et leur profond sentiment religieux s'ancre dans la Terre d'Israël, dont la nature est indissociable :

Dites bien au président des États-Unis qu'il n'a pas la moindre chance contre nous ! Car le Roi de l'Univers lui-même nous soutient ! [...] Vous affaiblissez le peuple d'Israël de retour sur sa terre après deux mille ans d'exil, de persécutions, de pogroms et une tentative d'extermination! Et vous voulez nous forcer à partir d'ici, de ce Sanctuaire divin qui est la terre de nos ancêtres ?!. (324, 217-218)

Certes, Nahman de Bratslav pratiquait ses exercices de contemplation dans la nature en Galicie, et on peut supposer que de nombreux hassidim en diaspora font de même. Cependant, la Terre d'Israël occupe indéniablement une place importante dans la démarche des personnages. Certes, Neta Hirschzon qui prononce ces paroles est hors d'elle, mais on ne comprend pas pourquoi le traducteur a supprimé le répétition de "mikan» (d'ici), qui restitue bien l'importance extraordinaire que les implantés accordent au lieu. Mais par ailleurs, leur spiritualité n'est pas détachée de l'histoire, elle repose bien sur ses deux jambes: la Nature et l'Histoire, la Création et le Peuple d'Israël, autrement dit, ils vivent pleinement une religion qui unit les trois termes de l'Alliance : Dieu, la Nature et le Peuple.

Au terme de cet examen, il apparaît que l'articulation entre la Bible et la Nature, dans ce roman, se fait par des hommes qui ont à cœur de vivre pleinement les termes de l'Alliance conclue par le Créateur avec l'humanité, qui passe par l'étude et l'observation des commandements et par la rencontre avec Dieu au sein de sa Création. C'est pourquoi l'articulation entre Nature et Bible, dans le roman, est tout à fait cohérente; elle passe par la médiation de la sensibilité humaine, certes exacerbée dans ces milieux hassidiques, mais elle rétablit ainsi une relation qui, semble-t-il, était impliquée par le texte biblique: une

\footnotetext{
${ }^{10}$ Emmanuel Levinas, Du sacré au saint, Paris, éd. de Minuit, 1977.
} 
relation triangulaire entre le Créateur, la Création et les hommes, relation parfaitement restituée par l'auteur, appelant ceux-ci à être pleinement partenaires avec Lui.

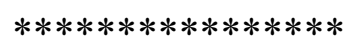

\section{BIBLIOGRAPHIE}

Instruments de travail :

- Bible, traduction TOB (Traduction Ecuménique de la Bible), utilisée pour les citations bibliques contenues dans l'article.

- Rabbi Nahman de Bratslav, Contes, trad. Laurent Cohen, Villeurbanne, Matanel, 2013.

- Schökel Luis Alonso, Manuel de poétique hébraïque, Bruxelles, Lessius, 2013.

Ouvrages utilisés :

- Chalier Catherine, L'Alliance avec la nature, Paris, Cerf, 1989.

- Green Arthur, La Sagesse dansante de Rabbi Nahman, Paris, Albin Michel, 2000.

- Ouaknin Marc-Alain, Ouvertures hassidiques, Paris, éd. J. Grancher, 1990.

- Ouaknin Marc-Alain, Tsimtsoum, Paris, Albin Michel, 1992.

- Wiesel Élie, Célébration hassidique, Paris, Seuil, 1972.

Site internet: Le site d'Assaf Gavron (http://www.assafgavron.com) donne de nombreux renseignements sur son œuvre. 